\title{
Microdialysis Sampling Method for Evaluation of Binding Kinetics of Small Molecules to Macromolecules
}

\author{
Hailin Wang, ${ }^{*, \dagger}$ Zhixin Wang, ${ }^{\dagger}$ Meiling Lu, ${ }^{\dagger}$ and Hanfa Zou ${ }^{\ddagger}$ \\ State Key Laboratory of Environmental Chemistry and Eco-toxicology, Research Center for Eco-Environmental Sciences, \\ Chinese Academy of Sciences, Beijing 100085, China, and National Chromatographic Research \& Analysis Center, Dalian \\ Institute of Chemical Physics, Chinese Academy of Sciences, Dalian 116023, China
}

Here we present an application of microdialysis sampling for evaluation of the binding kinetics of small molecules to macromolecules. It is label-free, and no immobilization of any interaction partner is required. The method was established by the coupling of a binding reaction with a membrane transport in a miniature and dynamic microdialysis sampling system. A theoretical model was established to describe the quantitative relationship between the binding kinetics of small ligands to macromolecules and the enhanced mass transport of small ligands and was applied to estimate the binding kinetics. To demonstrate the proof-of-principle, we examined the binding kinetics of an abundant plasma protein human serum albumin (HSA) and a representative drug ketoprofen as an example. The primary binding constant of ketoprofen to HSA was estimated as $1.63( \pm 0.12) \times 10^{6} \mathrm{M}^{-1}$. The estimated association and dissociation rate constants $\left(k_{1}\right.$ and $\left.k_{-1}\right)$ were about $3.71 \times 10^{5} \mathrm{M}^{-1} \mathrm{~s}^{-1}$ and $0.227 \mathrm{~s}^{-1}$, respectively. The results suggest a fast binding of ketoprofen to HSA and a fast dissociation of the formed complex, which are consistent with the reversible binding property of drug and HSA $\left(k_{-1}\right.$ in the order of $\left.\mathbf{s}^{-1}\right)$. This is the first report on binding-kinetics measurement using microdialysis sampling.

Affinity interactions and even their binding kinetics can play important roles in every aspect of various cells, including gene regulation, ${ }^{1}$ cellular metabolism, and cellular signal transduction. ${ }^{2}$ A number of methods have been developed for the evaluation of affinity interactions and their binding kinetics, including stoppedflow analysis, affinity chromatography, ${ }^{3-5}$ affinity and kinetic

\footnotetext{
* Corresponding author. Tel/Fax: 0086-10-62849600. E-mail: hlwang@ rcees.ac.cn.

$\dagger$ Research Center for Eco-Environmental Sciences.

* Dalian Institute of Chemical Physics.

(1) Wickiser, J. K.; Breaker, R. R.; Crothers, D. M. Mol. Cell 2005, 18, 4960.

(2) Berg, O. G.; von Hippel, P. H. Ann. Rev. Biophys. Biophys. Chem. 1985, 14, 131-160.

(3) Loun, B.; Hage, D. S. Anal. Chem. 1996, 68, 1218-1225.

(4) Moaddel, R.; Jozwiak, K.; Yamaguchi, R.; Wainer, I. W. Anal. Chem. 2005 $77,5421-5426$.

(5) Jozwiak, K.; Haginaka, J.; Moaddel, R.; Wainer, I. W. Anal. Chem. 2002 , $74,4618-4624$
}

capillary electrophoresis, ${ }^{6-15}$ and surface plasmon resonance (SPR). ${ }^{16-20}$ Despite this progress, the measurement of the fast binding kinetics of small molecules to macromolecules is still a challenge. The SPR method could be used to monitor the association and dissociation processes of complexes in real-time without the use of labels..$^{16-20}$ The disadvantages of SPR are the heterogeneity of the sensor surface and the diffusional limitation, ${ }^{18,19}$ which may result in $10-100$ times lower rate constants. ${ }^{19}$ The SPR biosensors respond more sensitively to the complexes of protein-protein, protein-peptide, and DNA-protein because of large mass changes upon complex formation and less sensitively to the complexes of small molecules and macromolecules because of negligible mass change in comparison with that of the involved macromolecules themselves. Due to this limitation, the SPR methods have been applied to measure the binding constants of small molecules and macromolecules rather than their binding kinetics, especially for the binding with a large dissociation rate constant $\left(\mathrm{s}^{-1}\right){ }^{17,19}$ Affinity capillary electrophoresis is another powerful bioanalytical technique. Because of high separation efficiency, fast speed, and minute sample consumption, it has been intensively studied for measurement of the binding constants and binding stoichiometry of various affinity interactions.$^{6-10}$ Recently, Krylov et al. further proposed and developed kinetic capillary

(6) Rundlett, R. L.; Armstrong, D. W. Electrophoresis 1997, 18, 2194-2202.

(7) Rundlett, R. L.; Armstrong, D. W. J. Chromatogr., A 1996, 721, 173-186.

(8) Bowser, M. T.; Kranack, A. R.; Chen, D. D. Y. Anal. Chem. 1998, 70, 10761084.

(9) Galbusera, C.; Thachuk, M.; De Lorenzi, E.; Chen, D. D. Y. Anal. Chem. 2002, 74, 1903-1914.

(10) Bose, S.; Hage, D. S. J. Chromatogr., B 1997, 697, 77-88

(11) Yang, P.; Whelan, R. J.; Jameson, E. E.; Kurzer, J. H.; Argetsinger, L. S.; Carter-Su, C.; Kabir, A.; Malik, A.; Kennedy, R. T. Anal. Chem. 2005, 77, $2482-2489$

(12) Krylov, S. N. Electrophoresis 2007, 28, 69-88

(13) Berezovski, M.; Krylov, S. N. J. Am. Chem. Soc. 2002, 124, 13674-13675.

(14) Berezovski, M.; Krylov, S. N. Anal. Chem. 2005, 77, 1526-1529.

(15) Drabovich, A.; Berezovski, M.; Krylov, S. N. J. Am. Chem. Soc. 2005, 127, 11224-11225.

(16) Nguyen, B.; Tanious, F. A.; Wilson, W. D. Methods 2007, 42, 150-161.

(17) Rich, R. L.; Hoth, L. R.; Geoghegan, K. F.; Brown, T. A.; LeMotte, P. K.; Simons, S. P.; Hensley, P.; Myszka, D. G. Proc. Natl. Acad. Sci. U.S.A. 2002 99, 8562-8567.

(18) Liu, X.; Song, D.; Tian, Y.; Liu, Z.; Zhang, H. Sens. Actuators, B 2006, 117 $188-195$.

(19) de Mol, N. J.; Plomp, E.; Fischer, M. J. E.; Ruijtenbeek, R. Anal. Biochem. 2000, 279, 61-70.

(20) Frostell-Karlsson, A.; Remaeus, A.; Roos, H.; Andersson, K.; Borg, P.; Hamalainen, M.; Karlsson, R. J. Med. Chem. 2000, 43, 1986-1992. 


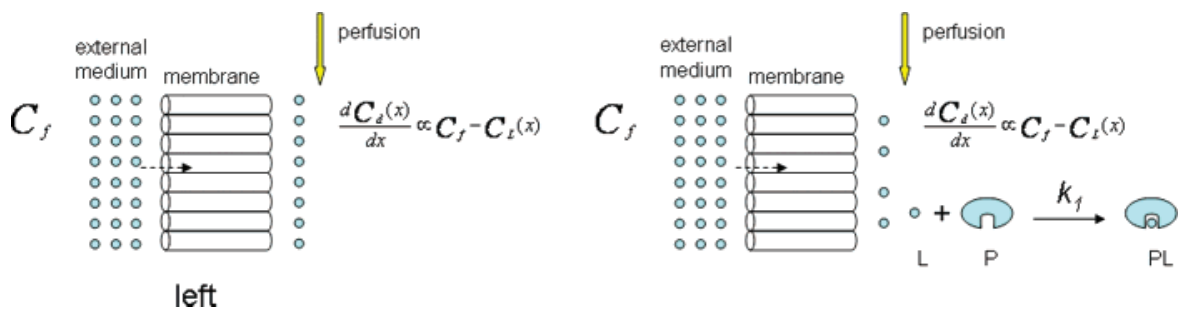

Figure 1. Schematic illustration of the mass transport of a small ligand (L) across membrane in the absence (left) and presence (right) of a large impermeable receptor protein $(P)$ in the perfusion fluid of microdialysis sampling. The decrease in concentration of free $L$ in the lumen of the probe and resultant enhancement of $L$ transport are associated with the binding kinetics of $L$ and $P$.

electrophoresis. ${ }^{12-16}$ Ideally, equilibrium constants and rate constants of DNA-protein interactions can be determined in one single experiment using kinetic capillary electrophoresis. It can measure the binding kinetics in a magnitude of minutes when a conventional capillary ( $20-40 \mathrm{~cm}$ capillary) is used. It is also possible to measure more rapid binding kinetics $\left(k_{-1}>2 \mathrm{~s}^{-1}\right)$ when an ultrafast separation $(4-8 \mathrm{~s})$ is achieved..$^{11}$ In this case, a unique flow-gated capillary electrophoresis laser-induced fluorescence has been used. ${ }^{11}$ However, successful affinity (and kinetic) capillary electrophoresis analysis requires sufficient mobility difference for reasonable resolution of the formed complex and free ligand.

Here, we present a novel method for the evaluation of the binding kinetics of small molecules to macromolecules using microdialysis sampling. This technology has some distinguished advantages. It takes advantage of the large size difference between small molecules and macromolecules and does not require any labeling, immobilization, mobility difference, or stopped flow. Compared with SPR instruments and methods, which only respond to the common property of refractive index or mass changes at the biospecific sensor surface upon complex formation, ${ }^{16}$ it can be hyphenated with a variety of powerful analytical techniques, e.g., capillary electrophoresis, liquid chromatography, mass spectrometry, and various biosensors. The developed microdialysis method can measure the binding kinetics in a magnitude of seconds, which is comparable to that of fast kinetic capillary electrophoresis. ${ }^{11,12}$ Unlike SPR and capillary electrophoresis, it does not require any expensive instrument and only requires an inexpensive injection pump and a microdialysis probe.

Microdialysis has been widely applied to in vivo monitoring of various bioactive compounds for studying physiological, pharmacological, and pathological changes because it only samples small molecules without the need to remove proteins. ${ }^{21}$ However, the recovery of microdialysis sampling is often very low. Continuous attempts have been made to improve its recovery. ${ }^{22-26}$ One way is to add an affinity trapping agent to microdialysis perfusion fluid serving to increase overall mass flux. ${ }^{22-26}$ Basically, a perfused affinity trapping agent can associate with the ligand of target in the perfusion fluid and cause the free concentration of the ligand to decrease at the inner membrane lumen, resulting in an increase

(21) Bourne, J. A. Clin. Exp. Pharmacol. Physiol. 2003, 30, 16-24.

(22) Stahle, L. Adv. Drug Delivery Rev. 2000, 45, 149-167.

(23) Davies, M. I.; Cooper, J. D.; Desmond, S. S.; Lunte, C. E.; Lunte, S. M. Adv. Drug Delivery Rev. 2000, 45, 169-188.

(24) Khramov, A. N.; Stenken, J. A. Anal. Chem. 1999, 71, 1257-1264.

(25) Duo, J.; Fletcher, H.; Stenken, J. A. Biosens. Bioelectron. 2006, 22, 449457.

(26) Khramov, A. N.; Stenken, J. A. Analyst 1999, 124, 1027-1033. in concentration gradient of the ligand across the membrane and an enhanced flux of the ligand. Albumin, cyclodextrins, antibody, and antibody-immobilized beads have been used as affinity trapping agents. ${ }^{24-27}$ In this work, we show the possibility of measuring the binding kinetics of small ligands and macromolecules by incorporating affinity trapping agents in perfusion fluid for microdialysis sampling.

Microdialysis sampling is essentially a dynamic and miniature variant of equilibrium dialysis and has been applied to study the interactions of small molecules and macromolecules, including those of drug-protein, drug-DNA, and metal-protein. ${ }^{28-34}$ However, it has not been applied to measure the binding kinetics. By taking advantage of the binding reaction-mediated transport across the semipermeable membrane and the miniaturization of the dialysis membrane, it is possible to measure the binding kinetics of small molecules to macromolecules. The miniaturization of dialysis membrane can reduce the volume of hollow membrane $(\sim$ hundred of nanoliters vs the milliliters of conventional dialysis, 10000 times lower) and diffusion distance (down to $50 \mu \mathrm{m}$ ) significantly, thereby eliminating the limitation caused by long-distance diffusion in conventional equilibrium dialysis $(\sim$ $10 \mathrm{~mm}$ ) and providing the possibility of conducting the measurement of binding kinetics. In this work, we demonstrate that the enhancement of microdialysis recovery by affinity trapping agents can be used to evaluate the binding kinetics using ketoprofen and HSA as an example. Ketoprofen is one of the nonsteroidal antiinflammatory drugs, highly bound to the abundant plasma protein HSA. The binding of ketoprofen to HSA and its kinetics together may affect the distribution, metabolism, and fate of keoprofen. The measurement of the binding of ketoprofen to HSA is a challenge because of its rapid dissociation.

\section{THEORETICAL SECTION}

Figure 1 illustrates the transport process of a small ligand (L) across a microdialysis membrane in the absence and presence of a large receptor protein $(\mathrm{P})$ in the perfusion fluid of microdialysis. The concentration of the permeable $\mathrm{L}$ is mainly determined by the concentration gradient of $\mathrm{L}$ across the membrane in the

(27) Carneheim, C.; Stahle, L. Pharmacol. Toxicol. 1991, 69, 378.

(28) Herrara, M.; Scott, D. O.; Lunte, C. E. Pharm. Res. 1990, 7, 1077.

(29) Ekblom, M.; Hammarlund, M.; Lundqvist. T.; Sjoberg, P. Pharm. Res. 1992 9, 155.

(30) Wang, H.; Zou, H.; Zhang, Y. Chromatographia 1997, 44, 205-208.

(31) Wang, H.; Zou, H.; Feng, A.; Zhang, Y. Anal. Chim. Acta 1997, 342, 159165.

(32) Wang, H.; Zou, H.; Zhang, Y. Anal. Chem. 1998, 70, 373-377.

(33) Guo, M.; Zou, H. F.; Wang, H. L.; Kong, L.; Ni, J. Y. Anal. Chim. Acta 2001, 443, 91-99.

(34) Tsai, T.-H. J. Chromatogr.. B 2003, 161-173. 
absence of $\mathrm{P}$ in the perfusion fluid. In this case, all the molecules of $\mathrm{L}$ are free (unbound). The total concentration of $\mathrm{L}$ in the microdialysates is not only determined by the concentration gradient of free $\mathrm{L}$ across the probe membrane but also affected by the association rate of $\mathrm{L}$ to $\mathrm{P}$ in the presence of impermeable $\mathrm{P}$ in the perfusion fluid. In both cases, the total concentration of L in the perfusion fluid $C_{\mathrm{d}}(\mathrm{M})$ is not changed over time at steady state; thus, a mass balance for $C_{\mathrm{d}}$ exists as

$$
J \Delta A+Q C_{\mathrm{d}, x}-Q C_{\mathrm{d}, x+\Delta x}=0
$$

where $x(\mathrm{~mm})$ represents the position of the perfusion fluid in the dialysis probe, $Q(\mu \mathrm{L} / \mathrm{min})$ is the volumetric perfusion flow rate through the microdialysis probe, and $J(\mathrm{M} \cdot \mathrm{mm} / \mathrm{min})$ and $\Delta A$ $\left(\mathrm{mm}^{2}\right)$ are the membrane permeate flux and the average area of the probe membrane with a length of $\Delta x(\mathrm{~mm})$, respectively. According to the definition of flux, ${ }^{35}$ eq 2 can be obtained as

$$
\begin{aligned}
J \Delta A=\frac{D_{\text {mem }}\left(C_{\mathrm{f}}-C_{\mathrm{L}}\right)}{\left(R_{\mathrm{o}}-R_{\mathrm{i}}\right)} \frac{2 \pi\left(R_{\mathrm{o}}-R_{\mathrm{i}}\right) \Delta x}{\ln \left(R_{\mathrm{o}} / R_{\mathrm{i}}\right)}= \\
\frac{2 \pi D_{\text {mem }}\left(C_{\mathrm{f}}-C_{\mathrm{L}}\right)}{\ln \left(R_{\mathrm{o}} / R_{\mathrm{i}}\right)} \Delta x
\end{aligned}
$$

where $C_{\mathrm{L}}(\mathrm{M})$ represents the free (unbound) concentration of $\mathrm{L}$ in the perfusion fluid, $D_{\text {mem }}\left(\mathrm{mm}^{2} / \mathrm{s}\right)$ is the diffusion coefficient of $\mathrm{L}$ through the porous, polymeric dialysis membrane, $C_{\mathrm{f}}(\mathrm{M})$ is the free concentration of $\mathrm{L}$ in the bulk solution of external medium, and $R_{0}$ and $R_{\mathrm{i}}(\mathrm{mm})$ are the outer and inner radii of the probe membrane, respectively. By substituting eq 2 into eq 1 , eq 1 can be transformed into eq 3 as below,

$$
\frac{\mathrm{d} C_{\mathrm{d}}}{\mathrm{d} x}=\frac{2 \pi D_{\text {mem }}}{\ln \left(R_{\mathrm{o}} / R_{\mathrm{i}}\right)} \frac{\left(C_{\mathrm{f}}-C_{\mathrm{L}}\right)}{Q}
$$

Given $\beta=\left\{2 \pi D_{\text {mem }}\right\} /\left\{\ln \left(R_{0} / R_{\mathrm{i}}\right)\right\}$, eq 3 can be simply rewritten as

$$
\frac{\mathrm{d} C_{\mathrm{d}}}{\mathrm{d} x}=\frac{\beta}{Q}\left(C_{\mathrm{f}}-C_{\mathrm{L}}\right)
$$

When $\mathrm{P}$ (a large receptor) with a total concentration of $C_{\mathrm{P}}(\mathrm{M})$ is introduced into the perfusion fluid (Figure 1, right), a reaction between $\mathrm{P}$ and L (assumed as 1:1 stoichiometry) can be described as follows:

$$
\mathrm{P}+\mathrm{L} \stackrel{k_{1}}{\longrightarrow} \mathrm{P} \cdot \mathrm{L}
$$

This is a second-order reaction. $k_{1}\left(\mathrm{M}^{-1} \mathrm{~s}^{-1}\right)$ is a second-order kinetic rate constant. If this reaction is a rate-limiting step in the protein binding coupled transport process, the initial rate of generating bound $\mathrm{L}$ is equal to $k_{1} C_{\mathrm{P}} C_{\mathrm{L}}$. The bound concentration of $\mathrm{L}, C_{\mathrm{b}}$, does not change over time at steady state; thus, a mass balance for $C_{\mathrm{b}}$ can be obtained as follows:

(35) Stenken, J. A.; Topp, E. M.; Southard, M. Z.; Lunte, C. E. Anal. Chem. 1993, $65,2324-2328$.

$$
k_{1} C_{\mathrm{P}} C_{\mathrm{L}} \pi R_{\mathrm{i}}^{2} \Delta x+Q C_{\mathrm{b}, x}-Q C_{\mathrm{b}, x+\Delta x}=0
$$

At the left side of eq 6 , the first item is the increased amount of bound $\mathrm{L}$ per unit time in the volume of $\pi R_{\mathrm{i}}^{2} \Delta x$ resulting from the predominant association of $\mathrm{L}$ with $\mathrm{P}$. Equation 6 can be further converted into eq 7 ,

$\frac{\mathrm{d} C_{\mathrm{b}}}{\mathrm{d} x}=\frac{\pi R_{\mathrm{i}}^{2}}{Q} k_{1} C_{\mathrm{P}} C_{\mathrm{L}}=\frac{\alpha}{Q} C_{\mathrm{P}} C_{\mathrm{L}} \quad\left(\right.$ provided $\left.\alpha=\pi R_{\mathrm{i}}^{2} k_{1}\right)$

The change of free concentration of $\mathrm{L}$ in the perfusion fluid $\left(C_{\mathrm{L}}\right)$ equals the difference in the change of $C_{\mathrm{d}}$ and $C_{\mathrm{b}}$ along $x$; thus

$$
\frac{\mathrm{d} C_{\mathrm{L}}}{\mathrm{d} x}=\frac{\mathrm{d} C_{\mathrm{d}}}{\mathrm{d} x}-\frac{\mathrm{d} C_{\mathrm{b}}}{\mathrm{d} x}=\frac{\beta}{Q}\left(C_{\mathrm{f}}-C_{\mathrm{L}}\right)-\frac{\alpha}{Q} C_{\mathrm{P}} C_{\mathrm{L}}
$$

Equation 8 can be rewritten as eq 9 ,

$$
\frac{\mathrm{d} C_{\mathrm{L}}}{\mathrm{d} x}+\frac{\beta+\alpha C_{\mathrm{P}}}{Q} C_{\mathrm{L}}=\frac{\beta C_{\mathrm{f}}}{Q}
$$

Equation 9 is an example of a linear equation with an initial boundary condition of at $x=0, C_{\mathrm{L}}=0$. In other words, the inlet concentration of $\mathrm{L}$ is zero, which is a valid initial value. Thereby, eq 9 can be solved as another equation:

$$
C_{\mathrm{L}}(x)=\frac{\beta C_{\mathrm{f}}}{\beta+\alpha C_{\mathrm{P}}}\left(1-\mathrm{e}^{-\left\{\left(\beta+\alpha C_{\mathrm{P}}\right) / Q\right\}}\right)
$$

Combining eqs 10 and 4 , an expression formula of $C_{\mathrm{d}}$ can be finally obtained as

$$
\begin{aligned}
C_{\mathrm{d}}(x)=\frac{\alpha \beta x}{Q\left(\beta+\alpha C_{\mathrm{P}}\right)} & C_{\mathrm{P}} C_{\mathrm{f}}+ \\
& \frac{\beta^{2}}{\left(\beta+\alpha C_{\mathrm{P}}\right)^{2}}\left(1-\mathrm{e}^{-\left\{\left(\beta+\alpha C_{\mathrm{P}}\right) / Q\right\} x}\right) C_{\mathrm{f}}
\end{aligned}
$$

This equation can be exploited to explain the influence of concentration of $\mathrm{P}\left(C_{\mathrm{P}}\right)$ and perfusion flow rate $Q$ on the microdialysis sampling recovery. By substituting eq 10 into eq 11 , then we obtain

$$
C_{\mathrm{d}}(x)=\frac{\alpha \beta x}{Q\left(\beta+\alpha C_{\mathrm{P}}\right)} C_{\mathrm{P}} C_{\mathrm{f}}+\frac{\beta}{\left(\beta+\alpha C_{\mathrm{P}}\right)} C_{\mathrm{L}}(x)
$$

When $\mathrm{P}$ is not added to the perfusion fluid $\left(C_{\mathrm{P}}=0\right)$, it is a conventional microdialysis sampling, and eq 12 is reduced as

$$
C_{\mathrm{d}}=C_{\mathrm{f}}\left(1-\mathrm{e}^{-\{\beta / Q\} x}\right)
$$

To facilitate nonlinear curve fitting analysis of $C_{\mathrm{d}}$ versus $C_{\mathrm{P}}$, eq 11 is rewritten as eq 14 : 


$$
\begin{aligned}
C_{\mathrm{d}}(x)=\frac{C_{\mathrm{P}}}{a+b C_{\mathrm{P}}}+ & \\
& \frac{b^{2} C_{\mathrm{f}}}{\left(b+a C_{\mathrm{P}}\right)^{2}}\left(1-\mathrm{e}^{-\left\{\left(1 /\left(a C_{\mathrm{f}}\right)\right)+\left(C_{\mathrm{P}} /\left(b C_{\mathrm{f}}\right)\right)\right\}}\right)
\end{aligned}
$$

In eq $14, a$ and $b$ are the nonlinear regression coefficients, equal to $\left\{Q /\left(\beta x C_{\mathrm{f}}\right)\right\}$ and $\left\{Q /\left(\alpha x C_{\mathrm{f}}\right)\right\}$, respectively. Since the coefficient $b=\left\{Q /\left(\alpha x C_{\mathrm{f}}\right)\right\}=\left\{Q /\left(\pi R_{\mathrm{i}}^{2} x k_{1} C_{\mathrm{f}}\right)\right\}$ and the internal volume of the probe membrane $V$ equals $\pi R_{\mathrm{i}}^{2} x$, the coefficient $b$ equals $(Q / V) /$ $\left(k_{1} C_{\mathrm{f}}\right) . V / Q$ is just the time that the perfusion fluid residing within the probe membrane $\left(t_{0}\right)$; thus, a formula for $k_{1}$ is achieved:

$$
k_{1}=\frac{1}{t_{0} C_{\mathrm{f}} b}
$$

From eq 15, the value of association rate constant $k_{1}$ can be estimated using the nonlinear regression coefficient $b$ and the measured free concentration of $\mathrm{L}$ in the external medium $\left(C_{\mathrm{f}}\right)$. Here, the regression coefficient $b$ is unitless.

It is also possible to derive the association rate constants by plotting microdialysis sampling recovery $(R)$ against the perfusion flow rate $(Q)$ according to eq 11 . Eq 11 can be transformed for nonlinear regression analysis as below:

$$
\begin{aligned}
R=\frac{C_{\mathrm{d}}(x)}{C_{\mathrm{f}}}= & \\
& \frac{a_{1} b_{1}}{Q\left(a_{1}+b_{1}\right)}+\frac{a_{1}^{2}}{\left(a_{1}+b_{1}\right)^{2}}\left(1-\mathrm{e}^{-\left\{\left(a_{1}+b_{1}\right)\right\} / Q}\right)
\end{aligned}
$$

In eq $16, a_{1}$ and $b_{1}$ are the nonlinear regression coefficiencts and equal $\beta x$ and $\alpha x C_{\mathrm{P}}$ (or $\pi R_{\mathrm{i}}^{2} x k_{1} C_{\mathrm{P}}$ ), respectively. The association rate constant can be derived from the coefficient $b_{1}$ according to eq 18:

$$
\begin{gathered}
b_{1}=\pi R_{\mathrm{i}}^{2} x k_{1} C_{\mathrm{P}}=V k_{1} C_{\mathrm{P}} \\
k_{1}=\frac{b_{1}}{V C_{\mathrm{P}}}
\end{gathered}
$$

In eq 18 , the unit of the regression coefficient $b_{1}$ is $\mu \mathrm{L} / \mathrm{min}$, the same as that used for the perfusion flow rate. The unit for $b_{1}$ can be easily inferred from eq 16 since the item $\left(b_{1} / Q\right)$ in eq 16 should be unitless.

From eq 6 to eq 15, we assumed that the binding reaction occurring in the inner lumen of the microdialysis membrane is a rate-limiting step in the binding reaction coupled membrane transport process, which determines the concentration gradient of the ligand $\mathrm{L}$ across the membrane to diffuse into the hollow microdialysis membrane. If the binding reaction is not a ratelimiting step but an equilibrium binding process, the free concentration of small ligand $\mathrm{L}\left(C_{\mathrm{L}}\right)$ in the perfusion fluid is much lower than that in external medium $\left(C_{\mathrm{f}}\right)$ when $\mathrm{L}$ is highly bound to a high-affinity receptor $\mathrm{P}$. Therefore, eq $4,\left(\mathrm{~d} C_{\mathrm{d}} / \mathrm{d} x\right)=(\beta / Q)$ $\left(C_{\mathrm{f}}-C_{\mathrm{L}}\right)$, can be approximated as $\left(\mathrm{d} C_{\mathrm{d}} / \mathrm{d} x\right) \approx(\beta / Q) C_{\mathrm{f}}$, and the microdialysis recovery should not change significantly and be

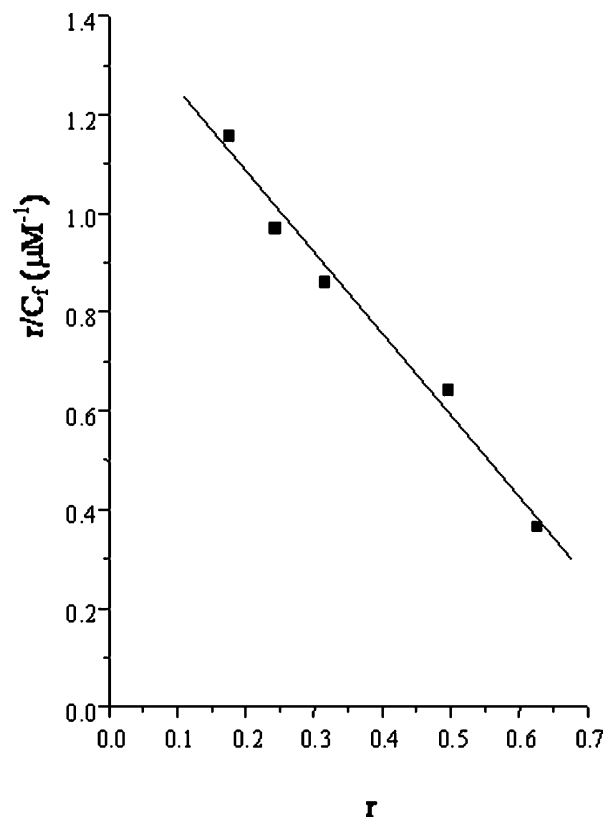

Figure 2. Scatchard plot for the noncovalent binding of KP to HSA.

independent from the concentration of the receptor $\left(C_{\mathrm{P}}\right)$. However, our work demonstrates that the enhanced microdialysis recovery is heavily dependent upon the concentration of the receptor $\mathrm{P}$ included in the perfusion fluid, which suggests that the association of $\mathrm{L}$ with the receptor $\mathrm{P}$ is a rate-limiting step (described in detail in the Results and Discussion).

\section{EXPERIMENTAL SECTION}

Chemicals. Racemic 2-(3-benzoylphenyl)-propionic acid (ketoprofen, KP) and human serum albumin (HSA, fatty acid and globulin-free) were purchased from Sigma (St. Louis, MO). The acetonitrile was of HPLC grade, and ultrapure water was prepared by a Milli-Q Ultrapure system. All other chemicals were of analytical grade.

Microdialysis. A CMA/20 microdialysis probe installed with a hollow dialysis membrane $(4 \mathrm{~mm}$ long, $0.5 \mathrm{~mm}$ o.d. and $0.4 \mathrm{~mm}$ i.d) was purchased from CMA/Microdialysis (Acton, MA). The membrane material is polycarbonate/polyether with a molecular weight cutoff of $20000 \mathrm{Da}$. The microdialysis probes were operated and preserved according to the manufacturer's instructions. A FAMILIC-100N microsyringe pump (JASCO, Kyoto, Japan) was used to pump the perfusion fluid through the microdialysis probe. The perfusion fluids were $0.067 \mathrm{M}$ potassium phosphate buffer ( $\mathrm{pH} 7.4)$ with an additive of HSA $(0-300 \mu \mathrm{M})$. Microdialysis sampling was carried out in a water bath at $35 \pm$ $0.2{ }^{\circ} \mathrm{C}$. The external solutions of the probe membrane were not stirred throughout the experiments.

Liquid Chromatography. The collected microdialysates were analyzed by HPLC-UV analysis. The chromatographic system consisted of a Waters 515 HPLC pump, a Waters 2487 UV detector (Waters, Milford, MA, U.S.A.), and a Hypersil BDS C18 column (5 $\mu \mathrm{m}, 200 \mathrm{~mm} \times 4 \mathrm{~mm}$ i.d., Dalian Elite analytical instrument, China). The mobile phase consisting of acetonitrile/water (23: $77, \mathrm{v} / \mathrm{v}$ ) and $10 \mathrm{mM}$ potassium phosphate buffer $(\mathrm{pH} 7.4)$ was filtered through a $0.45 \mu \mathrm{m}$ membrane and degassed by ultrasonication for $10 \mathrm{~min}$ before use. The flow rate was $0.8 \mathrm{~mL} / \mathrm{min}$. The 


\begin{tabular}{|c|c|c|c|c|c|}
\hline HSA added in perfusion fluid $(200 \mu \mathrm{M})$ & - & - & - & + & + \\
\hline $\begin{array}{l}\text { HSA added in external medium }(200 \mu \mathrm{M}) \\
\text { stirring in external medium } \\
\text { microdialysis recovery }(R, \%)\end{array}$ & $\begin{array}{l}- \\
- \\
49.9 \pm 0.7^{b}\end{array}$ & $\begin{array}{l}- \\
+ \\
57.5 \pm 1.5\end{array}$ & $\begin{array}{l}+ \\
- \\
71.0 \pm 2.0\end{array}$ & $\begin{array}{c}- \\
- \\
246.6 \pm 3.1\end{array}$ & $\begin{array}{l}+ \\
- \\
385.0 \pm 4.8\end{array}$ \\
\hline
\end{tabular}

analyte $\mathrm{KP}$ was detected at $259 \mathrm{~nm}$. The concentration of $\mathrm{KP}$ in microdialysates was estimated from a calibration curve constructed by a series of standard KP solutions $(0-40 \mu \mathrm{M})$. There was no significant measurement error for the peak area of KP whether HSA was present in microdialysates or not, and only the peak height of KP decreased slightly once a high concentration of HSA (up to $300 \mu \mathrm{M}$ ) was involved.

Free Concentration in External Medium and Microdialysis Sampling Recovery. The concentration ratio of the input analyte in the dialysate from an external medium to the free (unbound) analyte in the external medium is a measure of the recovery of the microdialysis sampling $(R)$. In the absence of HSA in the external medium, the recovery was directly determined by measuring the concentrations of the analyte in the microdialysate and in the external medium. In the presence of HSA in the external medium, the no net flux method ${ }^{36,37}$ was adopted for measuring the free analyte in the external medium and the recovery. In this work, the microdialysis sampling recovery and the free concentration of KP in the external medium were measured in the presence of HSA as described below in detail.

A $2.5 \mathrm{~mL}$ mixed solution of $\mathrm{KP}(0-128 \mu \mathrm{M})$ and HSA (200 $\mu \mathrm{M}$ ) in $0.067 \mathrm{M}$ phosphate buffer ( $\mathrm{pH} 7.4$ ) was prepared as an external medium, and a microdialysis probe was inserted in the solution. The perfusion fluid with an additive of KP at the concentrations of $0,1.12,2.38$, and $3.52 \mu \mathrm{M}$ was then perfused through the probe at a flow rate of $1.0 \mu \mathrm{L} / \mathrm{min}$. The total concentration of KP in the microdialysates was measured using HPLC-UV analysis as described above. Linear regression analysis was performed on a plot of net flux versus the concentration of the perfusate according to the no net flux method. ${ }^{36,37}$ The recovery of microdialysis sampling of $\mathrm{KP}$ was given by the obtained slope, and the concentration of free KP in the presence of HSA in the external medium was given by the $x$-axis intercept. When HSA presented in both external medium and perfusion fluid, the free concentration of KP in the external medium was first estimated using the same no net flux method described above, then the total concentration of KP in the HSA-containing microdialysates was directly measured using HPLC-UV analysis. The recovery was then calculated as the ratio of the total concentration of KP in the HSA-containing microdialysates to the free concentration of KP in the external medium.

Measurement of Binding Kinetics. To examine the binding kinetics, we investigated the enhancement of membrane transport by HSA of varying concentrations $(0-300 \mu \mathrm{M})$ in the perfusion fluid. In the external medium, constant concentrations for HSA

(36) Lonnroth, P.; Jannson, P. A.; Smith, U. Am. J. Physiol. 1987, 256, E250E255.

(37) Lindfors, N.; Amberg, G.; Ungerstedt, U. J. Pharmacol. Methods 1989, 22, $141-156$. of $200 \mu \mathrm{M}$ and $\mathrm{KP}$ of $128 \mu \mathrm{M}$ were included in $0.067 \mathrm{M}$ phosphate buffer $(\mathrm{pH} 7.4)$. HSA $(0-300 \mu \mathrm{M})$ was included in the perfusion buffer (0.067 M phosphate buffer, $\mathrm{pH}$ 7.4). The perfusion solution was pumped at $1.0 \mu \mathrm{L} / \mathrm{min}$. The concentrations of $\mathrm{KP}$ in the microdialysates $\left(C_{\mathrm{d}}\right)$ were directly determined by HPLC-UV analysis. The measured free concentration of KP in the external medium was about $2.5 \mu \mathrm{M}$, which was obtained from the estimation by the no net flux method. The obtained data were used to simulate the binding kinetics according to eqs 14 and 15.

We also investigated the effect of perfusion flow rate on the mass transport enhancement by HSA. The same external medium (200 $\mu \mathrm{M}$ HSA and $128 \mu \mathrm{M}$ KP) was used, and $123 \mu \mathrm{M}$ HSA was added to the perfusion solution. The microdialysis recovery was measured at different flow rates of 1.0, 2.0, 3.0, and $4.0 \mu \mathrm{L} / \mathrm{min}$. The obtained data were used for the simulation of the binding kinetics according to eqs 16 and 18 .

Measurement of Binding Constant. The concentration of thefree (unbound) KP can be determined as described previously. ${ }^{30-32}$ The obtained data were analyzed using Scatchard equation ${ }^{38}$

$$
\frac{r}{C_{\mathrm{f}}}=-K r+n K
$$

Where $r$ is the ratio of the bound concentration of KP to the total concentration of HSA, $K$ is the binding constant, and $n$ is the number of the binding sites. By plotting $r / C_{\mathrm{f}}$ against $r$, the binding constant $(K)$ and binding number $(n)$ can be derived.

\section{RESULTS AND DISCUSSION}

Binding Reaction and Diffusion-Based Microdialysis Transport. We first investigated the effect of binding reaction (in terms of association and dissociation) on the diffusion-based microdialysis transport to optimize the reaction kinetic study. The measured values of KP recovery in the absence and presence of HSA are listed in Table 1 . When HSA $(200 \mu \mathrm{M})$ was only added to the external medium, the obtained recovery was about $71.0 \%$, which is higher than that obtained in the absence of HSA (49.5$57.5 \%)$. The stirring of the external medium can improve the microdialysis recovery slightly over that without stirring in the absence of HSA in the external medium (57.5 vs $49.5 \%$ ). The enhancement of mass transport by HSA in the external medium may be explained by the fast dissociation of the bound KP from HSA, which results in continuous replenishment for the loss of the free KP caused by its transport across membrane and maintains the free KP constant from the external membrane surface to the bulk solution. Alternatively, HSA in the external medium can reduce the nonspecific adsorption of $\mathrm{KP}$ on the probe

(38) Scatchard, G. Ann. NY Acad. Sci. 1949, 51, 660-672. 
membrane, somehow resulting in recovery improvement. In the case where HSA is only present in the external medium, the capability of membrane transport is restricted by the accumulation of continuously input KP in the perfusion fluid, which reduces the concentration gradient of free KP across the membrane. When HSA $(200 \mu \mathrm{M})$ was added only to the perfusion fluid, a large increase in the recovery (246.6\%) was obtained, surpassing the limit of $100 \%$ recovery. This suggests that HSA included in the perfusion fluid enhances the transport of free KP across the membrane dramatically. Because only the free KP can diffuse into the perfusion fluid filled in the hollow microdialysis membrane, these penetrated KP molecules may react with HSA when they meet excess HSA molecules in the perfusion fluid. The occurrence of the reaction leads to predominance of the HSA complexed KP and eliminates the accumulation of free KP in the perfusion fluid, resulting in a significant decrease in free concentration of KP in the inner lumen of the membrane and enlargement of the concentration gradient of free KP across the membrane, thereby promoting the transport of free KP across the membrane. This also suggests that the association of KP with HSA that takes place in the inner lumen of the probe membrane facilitates the membrane transport of free KP more efficiently than the dissociation of KP from HSA that takes place outside of the membrane or inhibition of nonspecific adsorption by HSA. If HSA is added to both the external medium and perfusion fluid, it will combine the release of KP from the bound HSA in the external medium with the association of KP and HSA in the perfusion fluid. Thus, the combination should enhance the membrane transport more efficiently and allow the measurement of the reaction kinetics in the perfusion fluid more accurately. Indeed, it displays a maximum microdialysis recovery of $385.0 \%$ when HSA of $200 \mu \mathrm{M}$ was included in both the perfusion fluid and external medium, which is 0.56 -fold or 4.42-fold higher than that enhanced by HSA only presented in the perfusion fluid or in the external medium. These results suggest that the combination of the association reaction in the perfusion fluid with the dissociation in the external medium is an optimal way to examine the binding kinetics of KP and HSA. The enhanced membrane transport capability can be linked with the association rate in the perfusion fluid. Therefore, we adopted this method to measure the reaction kinetics in this work.

Binding Constants. Before derivation of their binding kinetics, the binding constant of HSA and KP was estimated using microdialysis sampling, as described previously. ${ }^{31,32}$ The Scatchard plot is illustrated in Figure 2. The obtained binding number and binding constant of $\mathrm{KP}$ are $0.88 \pm 0.07$ and $(1.63 \pm 0.12) \times 10^{6}$ $\mathrm{M}^{-1}$, respectively. The correlation coefficient of linear regression analysis is above 0.99 . These values agree well with those determined by the microdialysis/HPLC-based competitive binding method $^{32}$ and the continuous filtration method. ${ }^{39}$

Binding Kinetics. Before evaluation of the binding kinetics of KP and HSA, we investigated the dependence of membrane transport upon the concentration of HSA incorporated in the perfusion fluid. The resultant enhancement of the membrane transport (microdialysis recovery) of KP by the binding reaction of HSA and KP in the perfusion fluid is shown in Figure 3. It is evident that the enhancement of the microdialysis recovery of $\mathrm{KP}$ is dependent upon the concentration of HSA present in the

(39) Shibukawa, A.; Terakita, A.; He, J. Y. J. Pharm. Sci. 1992, 81, 710.

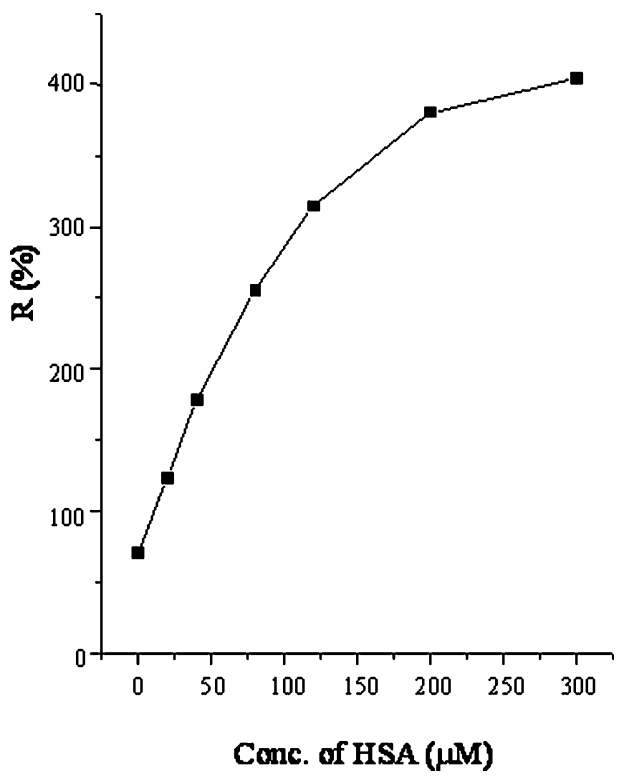

Figure 3. Influence of HSA concentration on the enhancement of microdialysis-based membrane transport. The external medium consisted of $128 \mu \mathrm{M} \mathrm{KP}$ and $200 \mu \mathrm{M}$ HSA in $0.067 \mathrm{M}$ phosphate buffer. The free concentration of KP is estimated by the no net flux method and is about $2.5 \mu \mathrm{M}$. The concentration of $\mathrm{KP}$ was repeatedly measured three times, and only the average values are shown. The measured relative standard error is less than $5 \%$.

perfusion fluid. The microdialysis recovery was over $400 \%$ and enhanced 5.70-fold once $300 \mu \mathrm{M}$ HSA was added to the perfusion fluid. According to the analysis described in the Theoretical Section, the dependence of microdialysis recovery upon the concentration of HSA in the perfusion fluid indicates that the enhancement of membrane transport is determined by the association rate rather than the binding equilibrium. Under investigated conditions, KP of over $90 \%$ is bound to HSA in the perfusion fluid with the concentration of HSA $(25-300 \mu \mathrm{M})$ if the binding is in equilibrium. In such a case, maximum concentration gradient could be maintained along the membrane, and there is no significant change in the concentration gradient on both sides of the microdialysis membrane in the presence of HSA with the concentration ranging from 25 to $300 \mu \mathrm{M}$. Therefore, the membrane transport should be enhanced in similar degree. The significant increase in membrane transport of KP with increasing concentration of HSA in the perfusion fluid supports that the binding kinetics plays a more important role in enhancement of membrane transport. Therefore, it is possible to measure the binding kinetics of KP and HSA by incorporating HSA in the prefusion fluid in microdialysis sampling.

As we discussed in the Theoretical Section, eq 14 was used to evaluate the reaction kinetics of KP-HSA binding. The obtained nonlinear curve of $C_{\mathrm{d}}$ against $C_{\mathrm{HSA}}$ is illustrated in Figure 4. A good correlation is obtained with nonlinear correlation coefficient (chi square) of $0.6896(n=6)$. The obtained coefficients of $a$ and $b$ are $7.54( \pm 0.83))$ and $0.0719( \pm 0.0051)$, respectively. The association rate constant can be estimated according to eq 15 as below.

$$
k_{1}=\frac{1}{t_{0} C_{\mathrm{f}} b}
$$




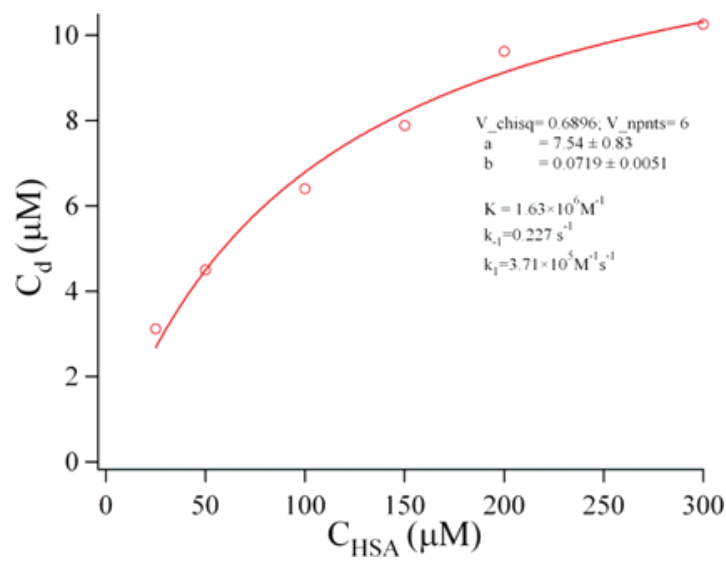

Figure 4. Binding kinetic analysis of KP and HSA by nonlinear regression of $C_{\mathrm{d}}$ against $C_{\mathrm{HSA}}$.

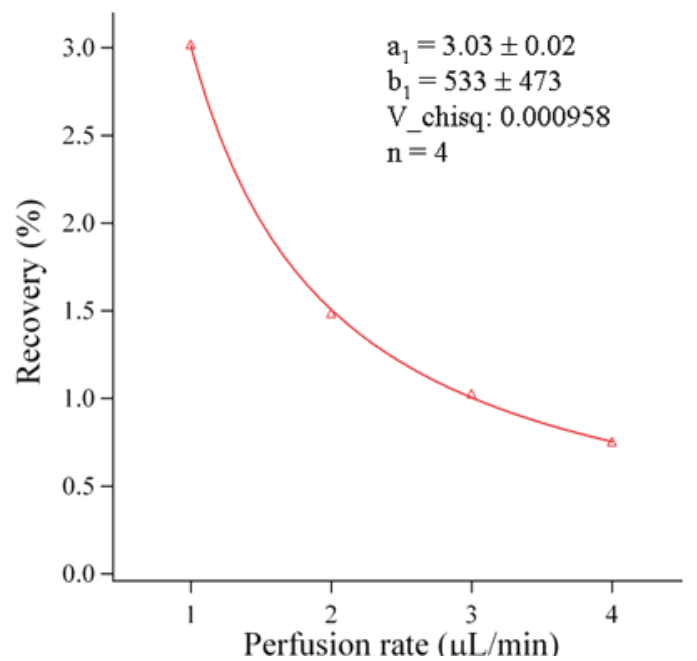

Figure 5. Binding kinetic analysis of KP and HSA by nonlinear regression of recovery $(R)$ against perfusion flow rate $(Q)$. The external medium was same as that described in Figure 3 . The perfusion solution contained $123 \mu \mathrm{M}$ HSA. The recovery was repeatedly measured three times, and only the average values are shown. The measured relative standard error is less than $5 \%$.

The internal volume of the microdialysis probe is about $0.25 \mu \mathrm{L}$, and the perfusion flow rate is $1.0 \mu \mathrm{L} / \mathrm{min}$; thus, the migration time $(t)$ through the probe is about $15 \mathrm{~s}$. The $C_{\mathrm{f}}$ value of $\mathrm{KP}$ in the assay is estimated as about $2.50 \mu \mathrm{M}$. By substituting the values of $t_{0}, C_{\mathrm{f}}$, and $A$ into the above equation, the estimated association constant $k_{1}$ is about $3.71 \times 10^{5} \mathrm{M}^{-1} \mathrm{~s}^{-1}$. From the measured association rate constant of KP and HSA $\left(K=1.63 \times 10^{6} \mathrm{M}^{-1}\right)$, the dissociation rate constant is estimated as about $0.227 \mathrm{~s}^{-1}$.

We further estimated the association rate constant by nonlinear regression analysis of recovery versus perfusion flow rate according to eq 16 (Figure 5). A good correlation is also obtained with the nonlinear correlation coefficient (chi square) of $0.001(n=$ 4). The obtained nonlinear regression coefficient $b_{1}$ is $553 \mu \mathrm{L} /$ min. According to eq $18, k_{1}=\left\{b_{1} /\left(V C_{\mathrm{P}}\right)\right\}$, the association rate constant $\left(k_{1}\right)$ of KP and HSA is estimated as $2.89 \times 10^{5} \mathrm{M}^{-1} \mathrm{~s}^{-1}$ with the known volume of the probe membrane $(V=0.25 \mu \mathrm{L})$ and the HSA concentration $(123 \mu \mathrm{M})$. The dissociation rate constant $k_{-1}$ can also be estimated from the obtained association rate constant and binding constant $\left(K=1.63 \times 10^{6} \mathrm{M}^{-1}\right)$, and it is $0.176 \mathrm{~s}^{-1}$.
The estimated rate constants by the two methods (recovery versus protein concentration and recovery versus perfusion flow rate) conform well to each other, demonstrating the possibility of measuring the binding kinetics of small molecules and macromolecules using microdialysis sampling. The obtained parameters of binding kinetics suggest a fast binding of KP to HSA and fast dissociation of the KP-HSA complex, which is consistent with the nature of the reversible binding of abundant plasma protein HSA and drugs $\left(k_{-1}, 0.05-2 \mathrm{~s}^{-1}\right.$; and $k_{1}, 10^{4}-10^{6}$ $\left.\mathrm{M}^{-1} \mathrm{~s}^{-1}\right)^{3}$

This is the first report on study of binding kinetics of small molecules and macromolecules using microdialysis sampling and provides a simple and inexpensive technology for measuring the binding kinetics of protein-drug. It will be promising to extend this method to the measurement of the binding kinetics of drugDNA, drug-liposome, metal-protein, and metal-DNA.

\section{ACKNOWLEDGMENT}

This work was supported by the grants from National Basic Research Program of China (973 program, No. 2007CB407305) and National Natural Science Foundation of China (No. 20737003, 20677066, and 20621703) to Dr. H. Wang.

Received for review October 6, 2007. Accepted February 14, 2008.

\section{APPENDIX}

$C_{b}$ - The bound concentration of the ligand $\mathrm{L}$ to the receptor $\mathrm{P}$ in perfusion fluid $(\mathrm{M}, \mathrm{mol} / \mathrm{L})$.

$C_{\mathrm{d}}$ - The total concentration of the ligand $\mathrm{L}$ in the lumen of the probe membrane $(\mathrm{M})$.

$C_{\mathrm{f}}-$ The free (unbound) concentration of the ligand $\mathrm{L}$ in the bulk solution of external medium (M).

$C_{\mathrm{L}}$ - The free (unbound) concentration of the ligand $\mathrm{L}$ in the lumen of the probe membrane $(\mathrm{M})$.

$C_{\mathrm{P}}-$ The total concentration of the receptor in the perfusion fluid (M).

$\Delta A$ - The average area of the probe membrane with a length of $\Delta x\left(\mathrm{~mm}^{2}\right)$.

$D_{\text {mem }}$ - The diffusion coefficient of the ligand $\mathrm{L}$ through the porous, polymeric dialysis membrane $\left(\mathrm{mm}^{2} / \mathrm{s}\right)$.

$J$ - The membrane permeate flux $(\mathrm{M} \cdot \mathrm{mm} / \mathrm{s})$.

$K$ - The binding constant.

$k_{1}$ - The second-order association rate constant of the ligand $\mathrm{L}$ to the receptor $\left(\mathrm{M}^{-1} \mathrm{~s}^{-1}\right)$.

$k_{-1}$ - The dissociation rate constant of the complex $[\mathrm{P} \cdot \mathrm{L}]\left(\mathrm{s}^{-1}\right)$.

$Q$ - The volumetric perfusion flow rate through the probe $(u \mathrm{~L} /$ $\min )$.

$R_{\mathrm{i}}$ - The inner radius of the probe membrane $(\mathrm{mm})$.

$R_{0}$ - The outer radius of the probe membrane (mm).

$R$ - Recovery of microdialysis sampling (\%).

$t_{0}$ - The residence time of the ligand $\mathrm{L}$ within the probe lumen (min).

$V$ - The internal volume of the probe membrane $(\mu \mathrm{L})$.

\section{AC702066X}

